\title{
LIMIT CYCLES OF CONTINUOUS AND DISCONTINUOUS PIECEWISE-LINEAR DIFFERENTIAL SYSTEMS IN $\mathbb{R}^{3}$
}

\author{
BRUNO R. DE FREITAS ${ }^{1}$, JAUME LLIBRE ${ }^{2}$ AND JOAO C. MEDRADO ${ }^{3}$
}

\begin{abstract}
We study the limit cycles of two families of piecewise-linear differential systems in $\mathbb{R}^{3}$ with two pieces separated by a plane $\Sigma$. In one family the differential systems are only continuous on the plane $\Sigma$, and in the other family they are only discontinuous on the plane $\Sigma$.

The usual tool for studying these limit cycles is the Poincaré map, but here we shall use recent results which extend the averaging theory to continuous and discontinuous differential systems.

All the computations have been checked with the algebraic manipulator mathematica.
\end{abstract}

\section{Introduction and statement of the main Results}

The study of piecewise linear differential systems essentially started with Andronov, Vitt and Khaikin [1] and still continues to receive attention by researchers. The continuous and discontinuous piecewise-linear differential systems plays an important role inside the nonlinear dynamical systems. First they appear in a natural way in nonlinear engineering models, where certain devices are accurately modeled by such differential systems, see for instance the books of di Bernardo, Budd, Champneys and Kowalczyk [3], and Simpson [28], and the survey of Makarenkov and Lamb [26], and the hundreds of references quoted in these last three works. Moreover these kind of differential systems are frequent in applications from electronic engineering and nonlinear control systems, where they cannot be considered as idealized models; they are also used in mathematical biology as well, see for instance $[7,29,30,31]$.

There are many studies of the limit cycles of continuous and discontinuous piecewise-linear differential systems in $\mathbb{R}^{2}$ with two pieces separated by a straight line, see for instance $[2,4,6,9,10,11,12,13,14,15,16,21,22$, $23,25,27]$. But there are few results about the limit cycles of continuous and discontinuous piecewise-linear differential systems in $\mathbb{R}^{3}$ with two pieces separated by a plane. The objective of this work is to study the limit cycles of some of these last systems.

2010 Mathematics Subject Classification. Primary 34C05, 34C07, 37G15.

Key words and phrases. non-smooth differential system, limit cycle, piecewise linear differential system. 
We consider perturbations of the linear differential system

$$
\begin{aligned}
& \dot{x}=-y, \\
& \dot{y}=x, \\
& \dot{z}=i x,
\end{aligned}
$$

with $(x, y, z) \in \mathbb{R}^{3}$ and $i$ a real parameter. The dot denotes derivative with respect to an independent variable $t$, usually called the time. Straightforward computations show that the solutions of (1) are all periodic with the exception of the $z$-axis which is filled with equilibria.

In this paper first we study the periodic solution of the following perturbed continuous piecewise linear differential system

$$
\begin{aligned}
& \dot{x}=-y+\varepsilon(a+b x+c y+d|z|), \\
& \dot{y}=x+\varepsilon(e+f x+g y+h|z|), \\
& \dot{z}=i x+\varepsilon(j+k x+l y+m|z|),
\end{aligned}
$$

of system (1) with two zones $z>0$ and $z<0$, where $a, b, c, d, e, f, g, h, j, l$ and $m$ are real parameters and the parameter $\varepsilon>0$ is sufficiently small. Changing $z$ by $-z$ if necessary, we always can assume that the parameter $i \geq 0$.

Our main result on the periodic solutions of the continuous piecewise linear differential system (2) is the following. This result is obtained using the extension of the classical averaging theory for smooth differential systems to continuous differential systems given in [20], see section 2 for more details.

Theorem 1. Using the averaging theory of first order for the continuous piecewise linear differential system (2) the following statements hold.

(a) For $\varepsilon \neq 0$ sufficiently small if $i>0$ system (2) has the periodic solution

$$
(x(t), y(t), z(t))=\left(r^{*} \cos t+O(\varepsilon), r^{*} \sin t+O(\varepsilon), z^{*}+i r^{*} \sin t+O(\varepsilon)\right),
$$

if

$$
\frac{(b+g) \pi}{2 h i} \in\left[-\frac{\pi}{2}, 0\right) \cup\left(0, \frac{\pi}{2}\right] \quad \text { and } \quad \frac{2 \pi(e i-j)}{4 i(m-h i)}>0 .
$$

In the proof of this statement we describe how to compute the values of $r^{*}$ and $z^{*}$ in function of the parameters of system (2).

(b) If $i=0$ system (2) has two periodic solutions

$$
\begin{aligned}
& (x(t), y(t), z(t))=\left(O_{1}(\varepsilon) \cos t+O\left(\varepsilon^{2}\right), O_{1}(\varepsilon) \sin t+O\left(\varepsilon^{2}\right), \pm j / m+O_{2}(\varepsilon)\right), \\
& \text { if }(b+g) m \neq 0, j / m<0 \text { and } \varepsilon \neq 0 \text { sufficiently small is such that } \\
& O_{1}(\varepsilon)>0 .
\end{aligned}
$$

Theorem 1 is proved in section 3 .

Many problems in physics, economics, biology and applied areas are modeled by discontinuous differential systems but there exist only few analytical techniques for studying their periodic solutions. In [19] the authors extended 
the averaging theory to discontinuous differential systems. An improvement of this result for a much bigger class of discontinuous differential systems is given in [18].

Applying these tools we also investigate the periodic solutions of the discontinuous piecewise linear differential system

$$
\begin{aligned}
& \dot{x}=-y+\varepsilon(a+b x+c y+d f(z)), \\
& \dot{y}=x+\varepsilon(e+f x+g y+h f(z)), \\
& \dot{z}=i x+\varepsilon(j+k x+l y+m f(z)),
\end{aligned}
$$

with two pieces defined by $f(z)=z+\operatorname{sign}(z)$ and

$$
\operatorname{sign}(z)=\left\{\begin{array}{rll}
1 & \text { if } \quad z>0 \\
-1 & \text { if } \quad z<0
\end{array}\right.
$$

We get the following result on the periodic solutions of the discontinuous piecewise linear differential system (3).

Theorem 2. Using the averaging theory of first order for the discontinuous piecewise linear differential system (3), the following statements hold.

(a) If $i>0$ and $\left|\frac{4 \pi(e i-j)}{h i-m}\right| \in(0,4 \pi]$, for $\varepsilon \neq 0$ sufficiently small system (3) has the periodic solution

$$
(x(t), y(t), z(t))=\left(r^{*} \cos t+O(\varepsilon), r^{*} \sin t+O(\varepsilon), z^{*}+i r^{*} \sin t+O(\varepsilon)\right) \text {, }
$$$$
\text { where } z^{*}=-\frac{i r^{*}}{4} \sqrt{16-\frac{\pi^{2}(b+g+h i)^{2}\left(r^{*}\right)^{2}}{h^{2}}} \text { if } \frac{4 \pi(e i-j)}{h i-m} \in(0,4 \pi]
$$$$
\text { and } z^{*}=\frac{i r^{*}}{4} \sqrt{16-\frac{\pi^{2}(b+g+h i)^{2}\left(r^{*}\right)^{2}}{h^{2}}} \text { if }-\frac{4 \pi(e i-j)}{h i-m} \in(0,4 \pi] \text {. }
$$

(b) If $i>0,\left|\frac{4 \pi(e i-j)}{h i-m}\right| \in\left(4 \pi, \gamma^{*}\right)$ and $h(b+g-h i)<0$ or $h(b+g+$ $3 h i)>0$, for $\varepsilon \neq 0$ sufficiently small system (3) has two periodic solutions given by

$$
(x(t), y(t), z(t))=\left(r_{1,2}^{*} \cos t+O(\varepsilon), r_{1,2}^{*} \sin t+O(\varepsilon), z_{1,2}^{*}+i r_{1,2}^{*} \sin t+O(\varepsilon)\right),
$$

where $z_{1,2}^{*}=-\frac{i r_{1,2}^{*}}{4} \sqrt{16-\frac{\pi^{2}(b+g+h i)^{2}\left(r_{1,2}^{*}\right)^{2}}{h^{2}}}$ if $\frac{4 \pi(e i-j)}{h i-m} \in$ $\left(4 \pi, \gamma^{*}\right)$ and $z_{1,2}^{*}=\frac{i r_{1,2}^{*}}{4} \sqrt{16-\frac{\pi^{2}(b+g+h i)^{2}\left(r_{1,2}^{*}\right)^{2}}{h^{2}}}$ if $-\frac{4 \pi(e i-j)}{h i-m} \in$ $\left(4 \pi, \gamma^{*}\right)$.

(c) If $i=0,(b+g) m \neq 0,\left|-\frac{j}{m}\right|>1$ and $\varepsilon \neq 0$ sufficiently small is such that $O_{1}(\varepsilon)>0$, the system (3) has one periodic solution

$$
\begin{gathered}
(x(t), y(t), z(t))=\left(O_{1}(\varepsilon) \cos t+O\left(\varepsilon^{2}\right), O_{1}(\varepsilon) \sin t+O\left(\varepsilon^{2}\right), z^{*}+O_{2}(\varepsilon)\right), \\
\text { where } z^{*}=\frac{-j-m}{m} \text { if }-\frac{j}{m}>1, \text { or } z^{*}=\frac{-j+m}{m} \text { if }-\frac{j}{m}<-1 .
\end{gathered}
$$


In the proof of this theorem we describe how to compute the values of $r^{*}$ and $\gamma^{*}$ in function of the parameters of system (3).

Theorem 2 is proved in section 4 .

\section{BASiC RESUlts ON THE AVERAGING THEORY}

For proving Theorems 1 and 2 we apply two recent results from the averaging theory, one for the continuous piecewise linear differential systems, and the other for the discontinuous piecewise linear differential systems. In this section we present these results and some remarks necessary for their applications.

2.1. Continuous piecewise linear differential systems. From Theorem $\mathrm{B}$ of [20] taking the $k$ which appears in its statement equal to 1 we get the next result.

Theorem 3. Consider the following differential system

$$
\dot{x}(t)=F_{0}(t, x)+\varepsilon F_{1}(t, x)+\varepsilon^{2} R(t, x, \varepsilon),
$$

where $F_{i}: \mathbb{R} \times D \rightarrow \mathbb{R}^{n}$ for $i=0,1$ and $R: \mathbb{R} \times D \times\left(-\varepsilon_{0}, \varepsilon_{0}\right) \rightarrow \mathbb{R}^{n}$ and for each $t \in \mathbb{R}$ the functions $F_{0}(t,.) \in C^{1}, F_{1}(t,.) \in C^{0}$ and $D_{x} F_{0}$ is locally Lipschitz in the second variable, and $R \in C^{0}$ and locally Lipschitz in the second variable. Moreover $D \subset \mathbb{R}^{n}$ is an open subset and $\varepsilon$ is a small parameter. Assume that there exists an open and bounded subset of $V$ with its closure $\bar{V} \subset D$ such that for each $z \in \bar{V}$, the solution $x(t, z)$ of the unperturbed system $\dot{x}(t)=F_{0}(t, x)$ is T-periodic and satisfies $x(0, z)=z$. Denote by $M_{z}(t)$ the fundamental matrix of the variational equation

$$
\dot{M}_{z}(t)=D_{x} F_{0}(t, x(t, z)) M_{z}(t),
$$

with $z \in V$ such that $M_{z}(0)$ is the identity. If $a \in V$ is a zero of the map $f: V \rightarrow \mathbb{R}^{n}$ defined by

$$
f(z)=\int_{0}^{T} M_{z}^{-1}(t) F_{1}(t, x(t, z)) d t
$$

and

$$
\operatorname{det}\left(D_{z} f(a)\right) \neq 0,
$$

then for $\varepsilon>0$ sufficiently small, system (4) has a $T$-periodic solution $x(t, \varepsilon)$ such that $x(0, \varepsilon) \rightarrow a$ when $\varepsilon \rightarrow 0$.

The next result was obtained in [5], for a definition on the Brouwer degree see [24].

Theorem 4. We consider the following differential system

$$
\dot{x}=\varepsilon F_{1}(t, x)+\varepsilon^{2} R(t, x, \varepsilon),
$$


where $F_{1}: \mathbb{R} \times D \rightarrow \mathbb{R}^{n}, R: \mathbb{R} \times D \times\left(-\varepsilon_{f}, \varepsilon_{f}\right) \rightarrow \mathbb{R}^{n}$ are continuous functions, T-periodic in the first variable, and $D$ is an open subset of $\mathbb{R}^{n}$. We define $f: D \rightarrow \mathbb{R}^{n}$ as

$$
f(z)=\int_{0}^{T} F_{1}(s, z) d s
$$

and assume that:

(i) $F_{1}$ and $R$ are locally Lipschitz with respect to $x$;

(ii) for $a \in D$ with $f(a)=0$, there exists a neighborhood $V$ of a such that $f(z) \neq 0$ for all $z \in \bar{V} \backslash\{a\}$ and $d_{B}(f, V, 0) \neq 0$.

Then, for $|\varepsilon|>0$ sufficiently small, there exists an isolated $T$-periodic solution $x(t, \varepsilon)$ of system $(7)$ such that $x(0, \varepsilon) \rightarrow a$ as $\varepsilon \rightarrow 0$.

From the proof of Theorem 4 it follows the next remark.

Remark 5. If $f: D \rightarrow \mathbb{R}^{n}$ be a $C^{1}$ function, with $f(a)=0$, where $D$ is an open subset of $\mathbb{R}^{n}$ and $a \in D$. Whenever a is a simple zero of $f$ (i.e. the Jacobian $\operatorname{det} D f(a) \neq 0)$, there exists a neighborhood $V$ of a such that $f(z) \neq 0$ for all $z \in \bar{V} \backslash\{a\}$. Then $d_{B}(f, V, 0) \in\{-1,1\}$.

2.2. Discontinuous piecewise linear differential systems. Let $D \subset \mathbb{R}^{n}$ be an open subset and $h: \mathbb{R} \times D \rightarrow \mathbb{R}$ a $C^{1}$ function having 0 as regular value. Consider $F^{1}, F^{2}: \mathbb{R} \times D \rightarrow \mathbb{R}^{n}$ continuous functions and $\Sigma=h^{-1}(0)$. We define the discontinuous diffeential system as

$$
\dot{x}(t)=F(t, x)= \begin{cases}F^{1}(t, x) & \text { if } \quad(t, x) \in \Sigma^{+}, \\ F^{2}(t, x) & \text { if } \quad(t, x) \in \Sigma^{-},\end{cases}
$$

where $\Sigma^{+}=\{(t, x) \in \mathbb{R} \times D: h(t, x)>0\}$ and $\Sigma^{-}=\{(t, x) \in \mathbb{R} \times D$ : $h(t, x)<0\}$. The manifold $\Sigma$ is divided in the closure of two disjoint regions, namely Crossing region $\left(\Sigma^{c}\right)$ and Sliding region $\left(\Sigma^{s}\right)$ where

$$
\begin{aligned}
& \Sigma^{c}=\left\{p \in \Sigma:\left\langle\nabla h(p),\left(1, F^{1}(p)\right)\right\rangle\left\langle\nabla h(p),\left(1, F^{2}(p)\right)\right\rangle>0\right\}, \\
& \Sigma^{s}=\left\{p \in \Sigma:\left\langle\nabla h(p),\left(1, F^{1}(p)\right)\right\rangle\left\langle\nabla h(p),\left(1, F^{2}(p)\right)\right\rangle<0\right\} .
\end{aligned}
$$

The differential system (9) can be written as

$$
\dot{x}(t)=F(t, x)=\chi_{+}(t, x) F^{1}(t, x)+\chi_{-}(t, x) F^{2}(t, x),
$$

where $\chi_{+}, \chi_{-}$are the characteristic functions defined as

$$
\chi_{+}(t, x)=\left\{\begin{array}{ll}
1 & \text { if } \quad h(t, x)>0, \\
0 & \text { if } \quad h(t, x)<0,
\end{array} \text { and } \quad \chi_{-}(t, x)=\left\{\begin{array}{lll}
0 & \text { if } & h(t, x)>0, \\
1 & \text { if } & h(t, x)<0 .
\end{array}\right.\right.
$$

We apply the Fillipov's convention for the solutions of systems (9) or (10) passing through a point $(t, x) \in \Sigma$ (see [8]). Let $P$ be the space formed by the periodic solutions of (9) or (10). If $\operatorname{dim}(P)=\operatorname{dim}(D)=d$ then the following result follows directly from Theorem B of [18].

Theorem 6. Consider the differential system

$$
\dot{x}(t)=F_{0}(t, x)+\varepsilon F_{1}(t, x)+\varepsilon^{2} R(t, x, \varepsilon),
$$


where

$$
\begin{gathered}
F_{i}(t, x)=\chi_{+}(t, x) F_{i}^{1}(t, x)+\chi_{-}(t, x) F_{i}^{2}(t, x), \text { for } i=0,1, \text { and } \\
R(t, x)=\chi_{+}(t, x) R^{1}(t, x)+\chi_{-}(t, x) R^{2}(t, x),
\end{gathered}
$$

with $F_{i}^{1}, F_{i}^{2} \in C^{1}$, for $i=0,1$ and $R^{1}, R^{2}$ are continuous functions which are Lipschitz in the second variable, and all these functions are $T$-periodic functions in the variable $t \in \mathbb{R}$.

For $z \in D$ and $\varepsilon>0$ sufficiently small denote by $x(., z, \varepsilon):\left[0, t_{(z, \varepsilon)}\right] \rightarrow \mathbb{R}^{d}$ the solution of system (11) such that $x(0, z, \varepsilon)=z$. Define the averaged function

$$
f(z)=\int_{0}^{T} M_{z}^{-1}(s) F_{1}(s, x(s, z, 0)) d s,
$$

where $x(s, z, 0)$ is a periodic solution of (11) with $\varepsilon=0$ such that $x(0, z, 0)=$ $z$ and $M_{z}(s)$ is the fundamental matrix of the variational system $\dot{M}_{z}(t)=$ $D_{x} F_{0}(t, x(t, z, 0)) M_{z}(t)$ associated to the unperturbed system evaluated on the periodic solution $x(t, z, 0)$ such that $M_{z}(0)=I d$. Moreover we assume the following hypotheses.

$\left(H_{-}\right)$There exists an open bounded subset $C \subset D$ such that, for $\varepsilon$ sufficiently small, every orbit starting in $C$ reaches the set of discontinuity only at its crossing region.

$\left(H_{+}\right)$For $a \in C$ with $f(a)=0$ there exists a neighborhood $U \subset C$ of a such that $f(z) \neq 0$, for all $z \in \bar{U} /\{a\}$ and $\operatorname{det}\left(D_{z} f(a)\right) \neq 0$.

Then for $\varepsilon>0$ sufficiently small there exists a $T$-periodic solution $x(t, \varepsilon)$ of (11) such that $x(0, \varepsilon) \rightarrow a$ as $\varepsilon \rightarrow 0$.

Let $D$ be an open subset of $\mathbb{R}^{n}$. We shall denote the points of $\mathbb{R} \times D$. Let $h: \mathbb{R} \times D \rightarrow \mathbb{R}$ be a $C^{1}$ function having the $0 \in \mathbb{R}$ as a regular value, and let $\Sigma=h^{-1}(0)$.

Let $X, Y: \mathbb{R} \times D \rightarrow \mathbb{R}^{n}$ be two continuous vector fields. Assume that the functions $h, X$ and $Y$ are $T$-periodic in the variable $t$. Now we define a discontinuous piecewise differential system

$$
x^{\prime}(t)=Z(t, x)=\left\{\begin{array}{lll}
X(t, x) & \text { if } \quad h(t, x)>0 \\
Y(t, x) & \text { if } \quad h(t, x)<0 .
\end{array}\right.
$$

We concisely denote $Z=(X, Y)_{h}$.

The discontinuous differential system (12) can be written using the function $\operatorname{sign}(u)$ as

$$
x^{\prime}(t)=Z(t, x)=F_{1}(t, x)+\operatorname{sign}(h(t, x)) F_{2}(t, x),
$$

where

$$
F_{1}(t, x)=\frac{1}{2}(X(t, x)+Y(t, x)) \quad \text { and } \quad F_{2}(t, x)=\frac{1}{2}(X(t, x)-Y(t, x)) .
$$

The next result is Theorem A of [19]. 
Theorem 7. We consider the following discontinuous differential system

$$
x^{\prime}(t)=\varepsilon F(t, x)+\varepsilon^{2} R(t, x, \varepsilon),
$$

with

$$
\begin{aligned}
& F(t, x)=F_{1}(t, x)+\operatorname{sign}(h(t, x)) F_{2}(t, x), \\
& R(t, x, \varepsilon)=R_{1}(t, x, \varepsilon)+\operatorname{sign}(h(t, x)) R_{2}(t, x, \varepsilon),
\end{aligned}
$$

where $F_{1}, F_{2}: \mathbb{R} \times D \rightarrow \mathbb{R}^{n}, R_{1}, R_{2}: \mathbb{R} \times D \times\left(-\varepsilon_{0}, \varepsilon_{0}\right) \rightarrow \mathbb{R}^{n}$ and $h:$ $\mathbb{R} \times D \rightarrow \mathbb{R}$ are continuous functions, T-periodic in the variable $t$ and $D$ is an open subset of $\mathbb{R}^{n}$. We also suppose that $h$ is a $C^{1}$ function having 0 as a regular value.

Define the averaged function $f: D \rightarrow \mathbb{R}^{n}$ as

$$
f(x)=\int_{0}^{T} F(t, x) d t .
$$

We assume the following conditions.

(i) $F_{1}, F_{2}, R_{1}, R_{2}$ and $h$ are locally Lipschitz with respect to $x$;

(ii) there exists an open bounded subset $C \subset D$ such that, for $|\varepsilon|>0$ sufficiently small, every orbit starting in $\bar{C}$ reaches the set of discontinuity only at its crossing regions.

(iii) for $a \in C$ with $f(a)=0$, there exist a neighbourhood $U \subset C$ of a such that $f(z) \neq 0$ for all $z \in \bar{U} \backslash\{a\}$ and $d_{B}(f, U, 0) \neq 0$.

Then, for $|\varepsilon|>0$ sufficiently small, there exists a T-periodic solution $x(t, \varepsilon)$ of system (14) such that $x(0, \varepsilon) \rightarrow a$ as $\varepsilon \rightarrow 0$.

If the function $f(x)$ in (15) is $C^{1}$ the Remark 5 works for it.

\section{Proof of Theorem 1}

Proof of statement (a) of Theorem 1. By assumption we have that $i \neq 0$. Changing to cylindrical coordinates $x=r \cos \theta, y=r \sin \theta, z=z$, the system (2) writes as

$$
\begin{aligned}
\dot{r}= & \varepsilon g_{1}(\theta, r, z), \\
\dot{\theta}= & 1+\frac{\varepsilon}{r}(\cos \theta(e+h|z|+f r \cos \theta)-\sin \theta(a+d|z|+ \\
& \left.(b-g) r \cos \theta)-c r \sin ^{2} \theta\right), \\
\dot{z}= & i r \cos \theta+\varepsilon(j+m|z|+k r \cos \theta+l r \sin \theta),
\end{aligned}
$$

where

$$
\begin{aligned}
g_{1}(\theta, r, z)= & \cos \theta(a+d|z|+b r \cos \theta)+\sin \theta(e+h|z|+ \\
& (c+f) r \cos \theta)+g r \sin ^{2} \theta,
\end{aligned}
$$

and taking $\theta$ as the new independent variable system (2) becomes

$$
\begin{aligned}
& r^{\prime}=\varepsilon g_{1}(\theta, r, z), \\
& z^{\prime}=i r \cos \theta+\varepsilon g_{2}(\theta, r, z),
\end{aligned}
$$


where the prime denotes derivative with respect to $\theta$, and

$$
\begin{aligned}
g_{2}(\theta, r, z)= & j+m|z|-f i r \cos ^{3} \theta+l r \sin \theta-i \cos ^{2} \theta(e+ \\
& h|z|+(g-b) r \sin \theta)+\cos \theta(k r+d i|z| \sin \theta+ \\
& i \sin \theta(a+c r \sin \theta)) .
\end{aligned}
$$

The unperturbed system is

$$
\begin{aligned}
& r^{\prime}=0, \\
& z^{\prime}=i r \cos \theta .
\end{aligned}
$$

For each $\left(r_{0}, z_{0}\right)$ the solution $\varphi\left(\theta,\left(r_{0}, z_{0}\right)\right)$ such that $\varphi\left(0,\left(r_{0}, z_{0}\right)\right)=\left(r_{0}, z_{0}\right)$ is $\varphi\left(\theta,\left(r_{0}, z_{0}\right)\right)=\left(r_{0}, z_{0}+i r_{0} \sin \theta\right)$, which is $2 \pi$-periodic for all $r_{0} \neq 0$. For $r_{0}=0$ the system has a straight line filed of equilibria.

Now the differential system (17) is in the normal form (4) with

$$
\begin{aligned}
& F_{0}(\theta,(r, z))=(0, i r \cos \theta), \\
& F_{1}(\theta,(r, z))=\left(g_{1}(\theta, r, z), g_{2}(\theta, r, z)\right),
\end{aligned}
$$

satisfying all the assumptions of Theorem 3. So we apply this theorem to system (17) and we must calculate the averaged function

$$
f\left(r_{0}, z_{0}\right)=\int_{0}^{2 \pi} M^{-1}(\theta) F_{1}\left(\theta,\left(r_{0}, z_{0}\right)\right) d \theta,
$$

where the fundamental matrix $M(\theta)$ of the variational equation (5) on the periodic $(r(\theta), z(\theta))=\left(r_{0}, z_{0}+i r_{0} \sin \theta\right)$ of system (17), satisfying that $M(0)$ is the identity matrix, is

$$
M_{\left(r_{0}, z_{0}\right)}(\theta)=M(\theta)=\left(\begin{array}{cc}
1 & 0 \\
i \sin \theta & 1
\end{array}\right) .
$$

In short we have

$$
f\left(r_{0}, z_{0}\right)=\left(f_{1}\left(r_{0}, z_{0}\right), f_{2}\left(r_{0}, z_{0}\right)\right)
$$

where

$$
\begin{aligned}
& f_{1}\left(r_{0}, z_{0}\right)=\int_{0}^{2 \pi} g_{1}\left(\theta, r_{0}, z_{0}+i r_{0} \sin \theta\right) d \theta \\
& f_{2}\left(r_{0}, z_{0}\right)=\int_{0}^{2 \pi}\left(g_{2}\left(\theta, r_{0}, z_{0}+i r_{0} \sin \theta\right)-i \sin \theta g_{1}\left(\theta, r_{0}, z_{0}+i r_{0} \sin \theta\right)\right) d \theta .
\end{aligned}
$$

For calculating these two integrals for a given $z_{0}$ and $r_{0}$ we need to know the sign of $S=z_{0}+i r_{0} \sin \theta$ in function of $\theta$, because in the functions $g_{1}$ and $g_{2}$ appears the expression $\left|z_{0}+i r_{0} \sin \theta\right|$. Thus we have to distinguish the following cases.

For $i>0$ we have that

(i) If $\frac{z_{0}}{i r_{0}}<-1$, then $S<0$ for all $\theta$. If $\frac{z_{0}}{i r_{0}}=-1$, then $S<0$ for all $\theta$, except for $\theta=\frac{\pi}{2}$ where takes the value 0 . 
(ii) If $\left|\frac{z_{0}}{i r_{0}}\right|<1$, then $S>0$ when $\theta \in\left(-\arcsin \frac{z_{0}}{i r_{0}}, \pi+\arcsin \frac{z_{0}}{i r_{0}}\right)$;

(iii) If $\frac{z_{0}}{i r_{0}}>1$, then $S>0$ for all $\theta$. If $\frac{z_{0}}{i r_{0}}=1$, then $S>0$ for all $\theta$, except for $\theta=\frac{3 \pi}{2}$ where takes the value 0 .

Case (i): $\frac{z_{0}}{i r_{0}} \leq-1$. In this case the averaged function (19) is

$$
f\left(r_{0}, z_{0}\right)=\left((b+g-h i) \pi r_{0}, 2 \pi\left(-e i+j+h i z_{0}-m z_{0}\right)\right),
$$

whose unique zero is $\left(r_{0}, z_{0}\right)=\left(0, \frac{e i-j}{h i-m}\right)$. This solution (when it exists) corresponds to the equilibrium $\left(x=0, y=0, z=\frac{e i-j}{h i-m}\right)$ of the initial system when $\varepsilon=0$, so the averaging theory in this case does not provide any periodic solution.

Case (ii): $\left|\frac{z_{0}}{i r_{0}}\right|<1$. In this case the averaged function $f\left(r_{0}, z_{0}\right)=\left(f_{1}\left(r_{0}, z_{0}\right)\right.$, $\left.f_{2}\left(r_{0}, z_{0}\right)\right)$ is

$$
\begin{aligned}
& f_{1}\left(r_{0}, z_{0}\right)=\int_{-\arcsin }^{\pi+\arcsin \frac{z_{0}}{i r_{0}}}\left(b r_{0} \cos ^{2} \theta+\left(z_{0}+i r_{0} \sin \theta\right)(d \cos \theta+h \sin \theta)+\right. \\
& \left.\cos \theta\left(a+(c+f) r_{0} \sin \theta\right)+\sin \theta\left(e+g r_{0} \sin \theta\right)\right) d \theta+ \\
& \int_{\pi+\arcsin }^{2 \pi-\arcsin } \frac{z_{0}}{i r_{0}}\left(b r_{0} \cos \theta^{2}-\left(z_{0}+i r_{0} \sin \theta\right)(d \cos \theta+h \sin \theta)+\right. \\
& \left.\cos \theta\left(a+(c+f) r_{0} \sin \theta\right)+\sin \theta\left(e+g r_{0} \sin \theta\right)\right) d \theta, \\
& f_{2}\left(r_{0}, z_{0}\right)=\int_{-\arcsin }^{\pi+\arcsin \frac{z_{0}}{i r_{0}}} \frac{z_{0}}{i r_{0}}\left(j-e i+(m-h i)\left(z_{0}+i r_{0} \sin \theta\right)+\right. \\
& \left.(k-f i) r_{0} \cos \theta+(l-g i) r_{0} \sin \theta\right) d \theta+ \\
& \int_{\pi+\arcsin }^{2 \pi-\arcsin } \frac{z_{0}}{i r_{0}}\left(j-e i-(m-h i)\left(z_{0}+i r_{0} \sin \theta\right)+\right. \\
& \left.(r-f i) r_{0} \cos \theta+(l-g i) r_{0} \sin \theta\right) d \theta .
\end{aligned}
$$


Computing these integrals we obtain

$$
\begin{aligned}
& f_{1}\left(r_{0}, z_{0}\right)=\pi(b+g) r_{0}+2 h\left(z_{0} \sqrt{1-\frac{z_{0}^{2}}{i^{2} r_{0}^{2}}}+i r_{0} \operatorname{arccsc}\left(\frac{i r_{0}}{z_{0}}\right)\right), \\
& f_{2}\left(r_{0}, z_{0}\right)=2 \pi(j-e i)+4(m-h i)\left(i r_{0} \sqrt{1-\frac{z_{0}^{2}}{i^{2} r_{0}^{2}}}+z_{0} \operatorname{arccsc}\left(\frac{i r_{0}}{z_{0}}\right)\right) .
\end{aligned}
$$

Subcase (ii.1): Assume that $h$ and $m-h i$ are not zero. Then for computing the solutions of the system $f_{1}\left(r_{0}, z_{0}\right)=f_{2}\left(r_{0}, z_{0}\right)=0$, we must solve the equivalent system

$$
\begin{aligned}
& \frac{z_{0}}{i r_{0}} \sqrt{1-\frac{z_{0}^{2}}{i^{2} r_{0}^{2}}}+\operatorname{arccsc}\left(\frac{i r_{0}}{z_{0}}\right)=\alpha=-\frac{(b+g) \pi}{2 h i}, \\
& r_{0}\left(\sqrt{1-\frac{z_{0}^{2}}{i^{2} r_{0}^{2}}}+\frac{z_{0}}{i r_{0}} \operatorname{arccsc}\left(\frac{i r_{0}}{z_{0}}\right)\right)=\beta=\frac{2 \pi(e i-j)}{4 i(m-h i)},
\end{aligned}
$$

or equivalently we must solve the system

$$
\begin{aligned}
& a(u)=u \sqrt{1-u^{2}}+\operatorname{arccsc} \frac{1}{u}=\alpha, \\
& b(u)=r_{0}\left(\sqrt{1-u^{2}}+u \operatorname{arccsc} \frac{1}{u}\right)=\beta,
\end{aligned}
$$

where $u=\frac{z_{0}}{i r_{0}}$ with $u \in[-1,1]$. We obtain that $a(1)=\frac{\pi}{2}$ and $a(-1)=$ $-\frac{\pi}{2}$. In Figure 1 there are the graphics of the functions $a(u)$ and $b(u) / r_{0}$ respectively.
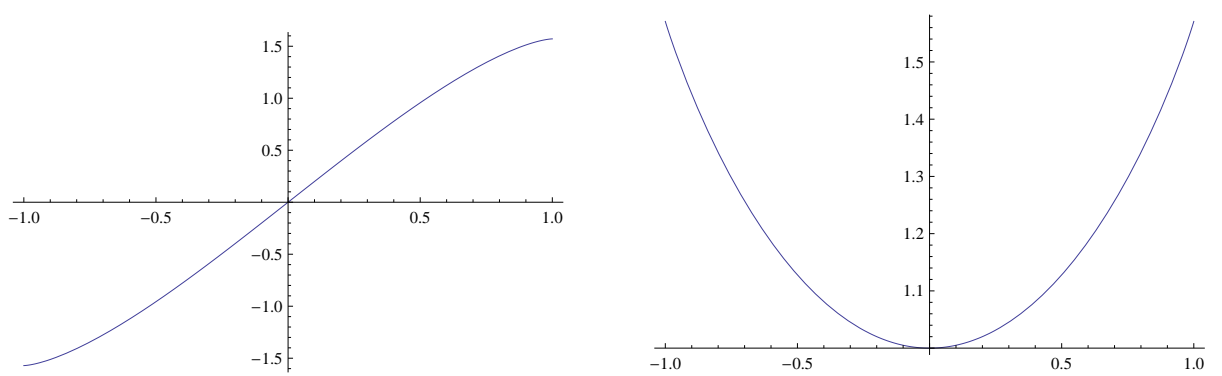

Figure 1. Graphics of functions $a(u)$ and $b(u) / r_{0}$.

From the graphic of $a(u)$ we conclude that given $\alpha \in[-\pi / 2, \pi / 2]$ there is a unique solution $u^{*} \in[-1,1]$ for $u$ satisfying the first equation of $(20)$. Substituting $u^{*}$ in second equation $b(u)=\beta$ of $(20)$, and taking into account the graphic of $b(u) / r_{0}$ it follows that there is a unique solution $r^{*}$ for $r_{0}>0$ if $\beta>0$ and $u^{*} \neq 0$. Hence we have proved that in the subcase (ii.1) 
there is one periodic solution using the averaging theory of first order if $\alpha \in[-\pi / 2,0) \cup(0, \pi / 2]$ and $\beta>0$. So, by Theorem 3, the periodic solution

$$
(r(\theta), z(\theta))=\left(r^{*}, z^{*}+i r^{*} \sin \theta\right)
$$

of the unperturbed system (1) can be continued to a periodic solution

$$
(r(\theta), z(\theta))=\left(r^{*}+O(\varepsilon), z^{*}+i r^{*} \sin \theta+O(\varepsilon)\right)
$$

of the perturbed system (17). Going back through the changes of coordinates this periodic solution for the system (16) becomes

$$
(r(t), \theta(t), z(t))=\left(r^{*}+O(\varepsilon), t+O(\varepsilon), z^{*}+i r^{*} \sin t+O(\varepsilon)\right) .
$$

Finally for system $(2)$ we get the periodic solution

$$
(x(t), y(t), z(t))=\left(r^{*} \cos t+O(\varepsilon), r^{*} \sin t+O(\varepsilon), z^{*}+i r^{*} \sin t+O(\varepsilon)\right) .
$$

In short we have proved the positive result of Theorem 1, but it remains to show that the averaging theory of first order does not provide more results on the periodic solutions of the continuous piecewise differential system (2).

Subcase (ii.2): Suppose that $h=0$ and $m-h i=0$. Then

$$
f_{1}\left(r_{0}, z_{0}\right)=\pi(b+g) r_{0} \text { and } f_{2}=2 \pi(j-e i) .
$$

This system has no solution if $j-e i \neq 0$. For $j-e i=0$ the Jacobian determinant (6) will be zero, so in any case the averaging theory does not provide information about the periodic solutions.

Subcase (ii.3): Suppose that $h=0$ and $m-h i \neq 0$. Therefore

$$
\begin{aligned}
& f_{1}\left(r_{0}, z_{0}\right)=\pi(b+g) r_{0}, \\
& f_{2}\left(r_{0}, z_{0}\right)=2 \pi(j-e i)+4(m-h i)\left(i r_{0} \sqrt{1-\frac{z_{0}^{2}}{i^{2} r_{0}^{2}}}+z_{0} \operatorname{arccsc}\left(\frac{i r_{0}}{z_{0}}\right)\right) .
\end{aligned}
$$

If $b+g=0$ then the Jacobian determinant (6) will be zero, so we can assume $b+g \neq 0$. Then we obtain the solution $r_{0}=0$, which corresponds to an equilibrium point of the unperturbed system (18), therefore the averaging theory does not provide any periodic solution in this subcase.

Subcase (ii.4): Suppose now that $h \neq 0$ and $m-h i=0$. We obtain

$$
\begin{aligned}
& f_{1}\left(r_{0}, z_{0}\right)=\pi(b+g) r_{0}+2 h\left(z_{0} \sqrt{1-\frac{z_{0}^{2}}{i^{2} r_{0}^{2}}}+i r_{0} \operatorname{arccsc}\left(\frac{i r_{0}}{z_{0}}\right)\right), \\
& f_{2}\left(r_{0}, z_{0}\right)=2 \pi(j-e i) .
\end{aligned}
$$

The same arguments than in the subcase (ii.2) show that the averaging theory does not provide information about the periodic solutions in this subcase. This completes the study of case (ii).

Case (iii): $\frac{z_{0}}{i r_{0}} \geq 1$. In this case the averaged function is given by

$$
f\left(r_{0}, z_{0}\right)=\left(\pi(b+g+h i) r_{0}, 2 \pi\left(j-e i+(m-h i) z_{0}\right)\right),
$$


whose unique zero is $\left(r_{0}, z_{0}\right)=\left(0, \frac{j-e i}{h i-m}\right)$. The conclusion follows as in Case (i).

Proof of statement (b) of Theorem 1. Since $i=0$ we have system (17) with $i=0$. This system satisfies the assumptions of the averaging Theorem 4 , so we must compute the function (23), i.e.

$$
\begin{aligned}
\left(f_{1}(r, z), f_{2}(r, z)\right)= & \int_{0}^{2 \pi}\left(g_{1}(\theta, r, z), g_{2}(\theta, r, z)\right) d \theta \\
& =(\pi(b+g) r, 2 \pi(j+m|z|)) .
\end{aligned}
$$

This function has two simple zeros if and only if $(b+g) m \neq 0$ and $j / m<0$, namely $(r, z)=(0, \pm j / m)$. Going back through the changes of variables as we did in the proof of statement (a) we get the result of statement (b).

We note that the function $\left(f_{1}(r, z), f_{2}(r, z)\right)$ has a unique zero if $(b+g) m \neq$ 0 and $j=0$, but in this case the Brouwer degree is zero, and the averaging Theorem 4 cannot be applied.

If $(b+g) m=0$, then it is easy to verify that the averaging Theorem 4 does not provide information about the periodic solutions of system (17). This completes the proof of statement (b) of Theorem 1 .

\section{Proof of Theorem 2}

Proofs of statements (a) and (b) of Theorem 2. Changing to cylindrical coordinates $x=r \cos \theta, y=r \sin \theta, z=z$, system (3) writes as

$$
\begin{aligned}
& \dot{r}= \varepsilon h_{1}(\theta, r, z), \\
& \dot{\theta}= 1+\frac{\varepsilon}{r}(\cos \theta(e+h z+f r \cos \theta+h \operatorname{sign}(z))-\sin \theta(a+d z+ \\
&\left.(b-g) r \cos \theta+d \operatorname{sign}(z))-c r \sin ^{2} \theta\right), \\
& \dot{z}=i r \cos \theta+\varepsilon(j+m z+k r \cos \theta+m \operatorname{sign}(z)+l r \sin \theta),
\end{aligned}
$$

where

$$
\begin{gathered}
h_{1}(\theta, r, z)=\cos \theta(a+d z+b r \cos \theta+d \operatorname{sign}(z))+\sin \theta(e+h z+ \\
(c+f) r \cos \theta+h \operatorname{sign}(z))+g r \sin ^{2} \theta,
\end{gathered}
$$

and taking $\theta$ as the new independent variable system (3) becomes

$$
\begin{aligned}
& r^{\prime}=\varepsilon h_{1}(\theta, r, z), \\
& z^{\prime}=i r \cos \theta+\varepsilon h_{2}(\theta, r, z),
\end{aligned}
$$


where the prime denotes derivative with respect to $\theta$, and

$$
\begin{aligned}
h_{2}(\theta, r, z)= & j+m z+\cos \theta(k r-i \cos \theta(e+h z+f r \cos \theta))+l r \sin \theta+ \\
& i \cos \theta \sin \theta(a+d z+(b-g) r \cos \theta+c r \sin \theta)+ \\
& \operatorname{sign}(z)(m+i \cos \theta(-h \cos \theta+d \sin \theta)) .
\end{aligned}
$$

As in the continuous case the unperturbed system is given by

$$
\begin{aligned}
& r^{\prime}=0, \\
& z^{\prime}=i r \cos \theta .
\end{aligned}
$$

Now the differential system (22) is in the normal form (11) with

$$
\begin{aligned}
& F_{0}(\theta,(r, z))=(0, i r \cos \theta), \\
& F_{1}(\theta,(r, z))=\left(h_{1}(\theta, r, z), h_{2}(\theta, r, z)\right),
\end{aligned}
$$

satisfying all the assumptions of Theorem 6 . So we apply this theorem to system (22) and we must calculate the averaged function

$$
f\left(r_{0}, z_{0}\right)=\left(f_{1}\left(r_{0}, z_{0}\right), f_{2}\left(r_{0}, z_{0}\right)\right)=\int_{0}^{2 \pi} M^{-1}(\theta) F_{1}\left(\theta,\left(r_{0}, z_{0}\right)\right) d \theta
$$

where as in the proof of statement (a) of Theorem 1 the fundamental matrix is

$$
M_{\left(r_{0}, z_{0}\right)}(\theta)=M(\theta)=\left(\begin{array}{cc}
1 & 0 \\
i \sin \theta & 1
\end{array}\right)
$$

In short we have

$$
\begin{aligned}
& f_{1}\left(r_{0}, z_{0}\right)=\int_{0}^{2 \pi} h_{1}\left(\theta, r_{0}, z_{0}+i r_{0} \sin \theta\right) d \theta \\
& f_{2}\left(r_{0}, z_{0}\right)=\int_{0}^{2 \pi}\left(h_{2}\left(\theta, r_{0}, z_{0}+i r_{0} \sin \theta\right)-i \sin \theta h_{1}\left(\theta, r_{0}, z_{0}+i r_{0} \sin \theta\right)\right) d \theta .
\end{aligned}
$$

Analogously to the study of the continuous system for $i>0$, we separate the computation of the averaged function in the same three cases, because in the functions $h_{1}$ and $h_{2}$ also appears the expression $\operatorname{sign}\left(z_{0}+i r_{0} \sin \theta\right)$.

Case (i): $\frac{z_{0}}{i r_{0}} \leq-1$. In this case the averaged function is

$$
f\left(r_{0}, z_{0}\right)=\left((b+g+h i) r_{0}, 2 \pi\left(-e i+j-m+m z_{0}+h i\left(1-z_{0}\right)\right)\right),
$$

whose unique zero is $\left(r_{0}, z_{0}\right)=\left(0, \frac{(h-e) i+j-m}{h i-m}\right)$. This solution (when it exists) corresponds to the equilibrium $(x, y, z)=\left(0,0, \frac{(h-e) i+j-m}{h i-m}\right)$ of the initial system when $\varepsilon=0$, so the averaging theory in this case does not provide any periodic solution. 
Case (ii): $\left|\frac{z_{0}}{i r_{0}}\right|<1$. In this case the averaged function $f\left(r_{0}, z_{0}\right)=\left(f_{1}\left(r_{0}, z_{0}\right)\right.$, $\left.f_{2}\left(r_{0}, z_{0}\right)\right)$ is

$$
\begin{aligned}
f_{1}\left(r_{0}, z_{0}\right)= & \int_{-\arcsin }^{\pi+\arcsin \frac{z_{0}}{i r_{0}}}\left(b r_{0} \cos ^{2} \theta+(d \cos \theta+h \sin \theta)+\cos \theta\left(a+d z_{0}+\right.\right. \\
& \left.\left.(c+f+d i) r_{0} \sin \theta\right)+\sin \theta\left(e+h z_{0}+(g+h i) r_{0} \sin \theta\right)\right) d \theta+ \\
& \int_{\pi+\arcsin }^{2 \pi-\arcsin \frac{z_{0}}{i r_{0}}}\left(b r_{0} \cos ^{2} \theta-(d \cos \theta+h \sin \theta)+\cos \theta\left(a+d z_{0}+\right.\right. \\
& \left.\left.(c+f+d i) r_{0} \sin \theta\right)+\sin \theta\left(e+h z_{0}+(g+h i) r_{0} \sin \theta\right)\right) d \theta, \\
f_{2}\left(r_{0}, z_{0}\right)= & \int_{-\arcsin }^{\pi+\arcsin \frac{z_{0}}{i r_{0}}}\left(-e i+j-h i z_{0}+m z_{0}+(-f i+k) r_{0} \cos \theta+\right. \\
& \left.(-h i+m)+(l-i(g+h i-m)) r_{0} \sin \theta\right) d \theta+ \\
& \int_{\pi+\arcsin \frac{z_{0}}{i r_{0}}}^{2 \pi-\arcsin \frac{z_{0}}{i r_{0}}}\left(-e i+j-h i z_{0}+m z_{0}+(-f i+k) r_{0} \cos \theta-\right. \\
& \left.(-h i+m)+(l-i(g+h i-m)) r_{0} \sin \theta\right) d \theta .
\end{aligned}
$$

Computing these integrals we obtain

$$
\begin{aligned}
& f_{1}\left(r_{0}, z_{0}\right)=\pi(b+g) r_{0}+h\left(\pi i r_{0}+4 \sqrt{1-\frac{z_{0}^{2}}{i^{2} r_{0}^{2}}}\right), \\
& f_{2}\left(r_{0}, z_{0}\right)=2 \pi(j-e i)+(m-h i)\left(2 \pi z_{0}+4 \operatorname{arccsc}\left(\frac{i r_{0}}{z_{0}}\right)\right) .
\end{aligned}
$$

Subcase (ii.1) Assume that $h$ and $m-h i$ are not zero. Note that $f_{1}\left(r_{0}, z_{0}\right)=$ 0 if and only if $z_{0}= \pm \frac{i r_{0}}{4} \sqrt{16-\frac{\pi^{2}(b+g+h i)^{2} r_{0}^{2}}{h^{2}}}$. As $\left|\frac{z_{0}}{i r_{0}}\right|<1$, we get that $(b+g+h i)$ is nonzero. These zeros exist if and only if $r_{0} \in$ $\left(0,\left|\frac{4 h}{(b+g+h i) \pi}\right|\right)$.

Assume that $\frac{h}{b+g+h i}>0$ and $z_{0}=-\frac{i r_{0}}{4} \sqrt{16-\frac{\pi^{2}(b+g+h i)^{2} r_{0}^{2}}{h^{2}}}$. In this case $z_{0}<0$ and thus $-1<\frac{z_{0}}{i r_{0}}<0$. Substituting this $z_{0}$ in $f_{2}\left(r_{0}, z_{0}\right)$, 
we get that $f_{2}\left(r_{0}, z_{0}\right)=0$ if and only if $G\left(r_{0}\right)=\mu$ where

$$
G\left(r_{0}\right)=\pi i r_{0} \sqrt{16-\frac{\pi^{2}(b+g+h i)^{2} r_{0}^{2}}{h^{2}}}+8 \operatorname{arccsc}\left(\frac{4}{\sqrt{16-\frac{\pi^{2}(b+g+h i)^{2} r_{0}^{2}}{h^{2}}}}\right),
$$

and $\mu=\frac{4 \pi(e i-j)}{h i-m}$. Note that $G\left(r_{0}\right) \geq 0, G(0)=4 \pi, G\left(r_{0}\right) \rightarrow 0$ if $r_{0} \rightarrow \frac{4 h}{\pi(b+g+h i)}$, and

$$
G^{\prime}\left(r_{0}\right)=\frac{16 \pi h^{2} i r_{0}-2 \pi^{3} i(b+g+h i)^{2} r_{0}^{3}-8 h^{2} \pi \sqrt{\frac{(b+g+h i)^{2} r_{0}^{2}}{h^{2}}}}{h^{2} r_{0} \sqrt{16-\frac{\pi^{2}(b+g+h i)^{2} r_{0}^{2}}{h^{2}}}} .
$$

As $\frac{h}{b+g+h i}>0$, we obtain that the point (when it is real)

$$
r_{0}^{c}=\frac{2}{\pi} \sqrt{-\frac{h(b+g-h i)}{i(b+g+h i)^{2}}}
$$

is a maximum of $G\left(r_{0}\right)$. It exists if and only if $h(b+g-h i) \leq 0$. Let $G\left(r_{0}^{c}\right)=\gamma^{*}$ be the maximum value of $G\left(r_{0}\right)$. In Figure 2 there are the graphics of the function $G\left(r_{0}\right)$ for $h(b+g-h i)<0$ and $h(b+g-h i) \geq 0$ respectively.
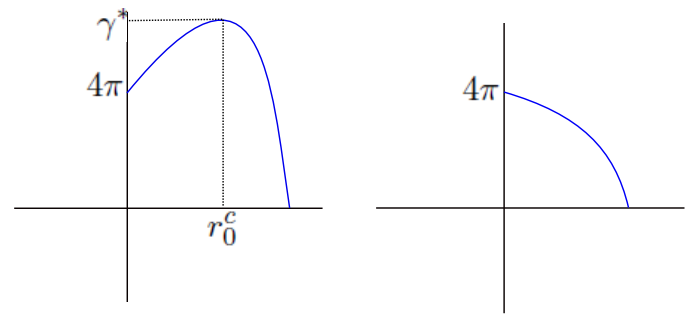

Figure 2. Function $G\left(r_{0}\right)$ for $h(b+g-h i)<0$ and $h(b+$ $g-h i) \geq 0$.

In short, from Theorem 6 , for $h(b+g-h i)<0$ we have that system $(22)$ has at least one periodic solution if $\mu \in(0,4 \pi]$, and system (22) has at least two periodic solutions if $\mu \in\left(4 \pi, \gamma^{*}\right)$.

For $h(b+g-h i) \geq 0$ the function $G\left(r_{0}\right)$ is decreasing and thus if $\mu \in(0,4 \pi)$, system (22) has exactly one periodic solution. In both cases going back through the changes of coordinates the periodic solutions of system (3) are of the form

$$
(x(t), y(t), z(t))=\left(r^{*} \cos t+O(\varepsilon), r^{*} \sin t+O(\varepsilon), z^{*}+i r^{*} \sin t+O(\varepsilon)\right),
$$


where $z^{*}=-\frac{i r^{*}}{4} \sqrt{16-\frac{(b+g+h i)^{2} \pi^{2}\left(r^{*}\right)^{2}}{h^{2}}}$, and $G\left(r^{*}\right)=\mu$.

For $\frac{h}{b+g+h i}<0$ we get analogous results, changing the critical point of function $G\left(r_{0}\right)$ (see $\left.(24)\right)$ for $\overline{r_{0}^{c}}=\frac{2}{\pi} \sqrt{\frac{h(b+g+3 h i)}{i(b+g+h i)^{2}}}$ that exists if and only if $h(b+g+3 h i) \geq 0$.

Suppose now that $z_{0}=\frac{i r_{0}}{4} \sqrt{16-\frac{(b+g+h i)^{2} \pi^{2} r_{0}^{2}}{h^{2}}}$. In this case $z_{0}>0$ and thus $0<\frac{z_{0}}{i r_{0}}<1$. Substituting $z_{0}$ in $f_{2}\left(r_{0}, z_{0}\right)$ we get that $f_{2}\left(r_{0}, z_{0}\right)=0$ if and only if $G\left(r_{0}\right)=-\mu$. The result is analogous to the previous case, changing $\mu$ by $-\mu$.

Subcase (ii.2): Suppose that $h=0$ and $m-h i=0$. The system $\left(f_{1}\left(r_{0}, z_{0}\right)\right.$, $\left.f_{2}\left(r_{0}, z_{0}\right)\right)=(0,0)$ has no solutions if $-e i+j \neq 0$, and when $-e i+j=0$ the Jacobian determinant (6) will be zero. So, by Theorem 6 in any case the averaging theory does not provide information about the periodic solution in this subcase.

Subcase (ii.3): Suppose that $h=0$ and $m-h i \neq 0$. We obtain the solution $r_{0}=0$, so the averaging theory does not provide periodic solution.

Subcase (ii.4): Suppose that $h \neq 0$ and $m-h i=0$. This case is similar to the subcase (ii.2). This completes the study of case (ii).

Case (iii): $\frac{z_{0}}{i r_{0}} \geq 1$. In this case the averaged function is given by

$$
f\left(r_{0}, z_{0}\right)=\left((b+g+h i) \pi r_{0}, 2 \pi\left(-e i+j+m+m z_{0}-h i\left(1+z_{0}\right)\right)\right),
$$

whose unique zero is $\left(r_{0}, z_{0}\right)=\left(0, \frac{(-e-h) i+j+m}{h i-m}\right)$. The conclusion follows as in Case (i).

Proof of statement (c) of Theorem 2. Since $i=0$ we have system (22) with $i=0$. This system satisfies the assumptions of the averaging Theorem 7 , so we must compute the function (15), i.e.

$$
\begin{aligned}
\left(f_{1}(r, z), f_{2}(r, z)\right)= & \int_{0}^{2 \pi}\left(h_{1}(\theta, r, z), h_{2}(\theta, r, z)\right) d \theta \\
& =(\pi(b+g) r, 2 \pi(j+m(z+\operatorname{sign}(z)))) .
\end{aligned}
$$

This function has one simple zero if and only if $(b+g) m \neq 0$, namely $(r, z)=$ $(0,(m-j) / m)$ if $-j / m<-1$ or $(r, z)=(0,(-m-j) / m)$ if $-j / m>1$. Going back through the changes of variables as we did in the proof of statement (a) we get the result of statement (b). 
Clearly if $(b+g) m=0$ the averaging Theorem 7 does not provide information about the periodic solutions. This completes the proof of statement (c) of Theorem 2.

\section{ACKNowledgments}

The first author is partially supported by the PROCAD-88881.068462/201401 and by the PRONEX/CNPq/FAPEG-2012.10.26.7000.803.

The second author is partially supported by a MINECO/FEDER grant MTM2008-03437 and MTM2013-40998-P, an AGAUR grant number 2014SGR568, an ICREA Academia, the grants FP7-PEOPLE-2012-IRSES 318999 and 316338, grant UNAB13-4E-1604, and a CAPES grant from the program CSF-PVE number 88881.030454/2013-01 .

The third author is partially supported by the FAPEG, by the CNPq grants numbers 475623/2013-4 and 306615/2012-6 and, by the CAPES grant numbers CSF/PVE-88881.030454/2013-01 and PROCAD-88881.068462/201401.

\section{REFERENCES}

[1] A. Andronov, A. Vitt and S. Khaikin, Theory of Oscillations, Pergamon Press, Oxford, 1966.

[2] J.C. Artés, J. Llibre, J.C. Medrado and M.A. Teixeira, Piecewise linear differential systems with two real saddles, Math. Comp. Sim. 95 (2013), 13-22.

[3] M. Di Bernardo, C. Budd, A. R. Champneys and P. Kowalczyk, Piecewisesmooth dynamical systems: theory and applications, Appl. Math. Sci. Vol. 163, Springer-Verlag, New York, 2007.

[4] D.C. Braga And L.F. Mello, Limit cycles in a family of discontinuous piecewise linear differential systems with two zones in the plane, Nonlinear Dynamics 73 (2013), $1283-1288$.

[5] A. Buica And J. Llibre, Averaging methods for finding periodic orbits via Brouwer degree, Bull. Sci. Math. 128 (2004), 7-22.

[6] C. Buzzi, C. Pessoa And J. Torregrosa, Piecewise linear perturbations of a linear center, Discrete Continuous Dyn. Syst. 9 (2013), 3915-3936.

[7] S. Coombes, Neuronal networks with gap junctions: A study of piecewise linear planar neuron models, SIAM Applied Dynamical Systems 7 (2008), 1101-1129.

[8] A.F. Filippov, Differential equations with discontinuous righthand sides, Mathematics and Its Applications, Kluwer Academic Publishers, Dordrecht, 1988.

[9] E. Freire, E. Ponce, F. Rodrigo and F. Torres, Bifurcation sets of continuous piecewise linear systems with two zones, Int. J. Bifurcation and Chaos 8 (1998), 2073 2097.

[10] E. Freire, E. Ponce And F. Torres, Canonical Discontinuous Planar Piecewise Linear Systems, SIAM J. Applied Dynamical Systems 11 (2012), 181-211.

[11] E. Freire, E. Ponce And F. Torres, A general mechanism to generate three limit cycles in planar Filippov systems with two zones, Nonlinear Dynamics 78 (2014), 251-263.

[12] F. Giannakopoulos And K. Pliete, Planar systems of piecewise linear differential equations with a line of discontinuity, Nonlinearity 14 (2001), 1611-1632.

[13] M. HAN AND W. Zhang, On Hopf bifurcation in non-smooth planar systems, J. of Differential Equations 248 (2010), 2399-2416. 
[14] S.M. HuAn AND X.S. YANG, On the number of limit cycles in general planar piecewise linear systems, Discrete and Continuous Dynamical Systems-A 32 (2012), 2147-2164.

[15] S.M. HuAn AND X.S. YANG, On the number of limit cycles in general planar piecewise linear systems of node-node types, J. Math. Anal. Appl. 411 (2013), 340-353.

[16] S.M. HuAN AND X.S. YANG, Existence of limit cycles in general planar piecewise linear systems of saddle-saddle dynamics, Nonlinear Anal. 92 (2013), 82-95.

[17] Y.T. LAU, The "cocoon" bifurcation in three-dimensional systems with two fixed points, Int. J. Bifurcation and Chaos 2 (1992), 543-558.

[18] J. Llibre AND D. NovaEs, On the periodic solutions of discontinuous piecewise differential systems, preprint, 2014.

[19] J. Llibre, D. Novaes and M.A. Teixeira, On the birth of limit cycles for nonsmooth dynamical systems, to appear in Bull. Sci. Math.

[20] J. Llibre, D. Novaes And M.A. Teixeira, Higher order averaging theory for finding periodic solutions via Brouwer degree, Nonlinearity 27 (2014), 563-583.

[21] J. Llibre, M. OrdóÑEz AND E. Ponce, On the existence and uniqueness of limit cycles in a planar piecewise linear systems without symmetry, Nonlinear Analysis Series B: Real World Applications 14 (2013), 2002-2012.

[22] J. Llibre And E. Ponce, Three nested limit cycles in discontinuous piecewise linear differential systems with two zones, Dynamics of Continuous, Discrete and Impulsive Systems, Serie B 19 (2012), 325-335.

[23] J. Llibre, M.A. Teixeira and J. Torregrosa, Lower bounds for the maximum number of limit cycles of discontinuous piecewise linear differential systems with a straight line of separation, Int. J. Bifurcation and Chaos 23 (2013), 1350066, pp. 10.

[24] N.G. Lloyd, Degree Theory, Cambridge University Press,1978.

[25] R. Lum, L.O. ChuA, Global properties of continuous piecewise-linear vector fields. Part I: Simplest case in $R^{2}$, Memorandum UCB/ERL M90/22, University of California at Berkeley, 1990.

[26] O. Makarenkov And J.S.W. Lamb, Dynamics and bifurcations of nonsmooth systems: A survey, Physica D 241 (2012), 1826-1844.

[27] S. Shui, X. Zhang AND J. LI, The qualitative analysis of a class of planar Filippov systems, Nonlinear Anal. 73 (2010), 1277-1288.

[28] D.J.W. Simpson, Bifurcations in Piecewise-Smooth Continuous Systems, World Scientific Series on Nonlinear Science A, vol 69, World Scientific, Singapore, 2010.

[29] R. Thul and S. Coombes, Understanding cardiac alternans: A piecewise linear modeling framework, Chaos 20 (2010), 045102-1-045102 13 pp.

[30] A. Tonnelier, The McKean's caricature of the Fitzhugh-Nagumo model I. The spaceclamped system, SIAM Journal of Applied Mathematics 63(2)(2003), 459-484.

[31] A. Tonnelier and W. Gerstner, Piecewise linear differential equations and integrate and-fire neurons: insights from two-dimensional membrane models, Phys. Rev. E 67 (2003), 021908, 16 pp.

1 Instituto de Matemática e Estatística, Universidade Federal de Goiás, 74001-970 Goî̂nia, Goiás, Brazil

E-mail address: freitasmat@ufg.br

2 Departament de Matemàtiques, Universitat Autònoma de Barcelona, 08193 Bellaterra, Barcelona, Catalonia, Spain

E-mail address: jllibre@mat.uab.cat

3 Instituto de Matemática e Estatística, Universidade Federal de Goiás, 74001-970 GoiÂNia, GoIÁs, BraziL

E-mail address: medrado@ufg.br 